\title{
Anti-beta 2 Glycoprotein I IgA in the SLICC Classification Criteria Dataset
}

Marwa Elkhalifa ${ }^{1}$, Ana-Maria Orbai ${ }^{2}$, Laurence S. Magder ${ }^{3}$ and Michelle Petri ${ }^{2}$

${ }^{1}$ Marwa Elkhalifa M.D. Department of Medicine, Alexandria University, School of Medicine, Alexandria, Egypt. Post-Doctoral Fellow, Johns Hopkins University School of Medicine.

${ }^{2}$ Ana-Maria Orbai M.D. M.H.S. Johns Hopkins University School of Medicine, Baltimore, MD, USA. 21205

${ }^{3}$ Laurence S. Magder M.P.H. Ph. D. Department of Epidemiology and Public Health, University of Maryland School of Medicine, Baltimore, MD, USA.

${ }^{2}$ Michelle Petri M.D. M.P.H. Professor, Johns Hopkins University School of Medicine,

Baltimore, MD, USA. 21205

\section{SLICC}

Graciela S Alarcón ${ }^{4}$, Caroline Gordon ${ }^{5}$, Joan Merrill ${ }^{6}$, Paul R Fortin 7 , Ian N Bruce ${ }^{8}$, David Isenberg $^{9}$, Daniel Wallace ${ }^{10}$, Ola Nived ${ }^{11}$, Rosalind Ramsey-Goldman ${ }^{12}$, Sang-Cheol Bae ${ }^{13}$, John G Hanly ${ }^{14}$, Jorge Sanchez-Guerrero ${ }^{15}$, Ann E Clarke ${ }^{16}$, Cynthia Aranow ${ }^{17}$, Susan Manzi ${ }^{18}$, Murray Urowitz ${ }^{15}$, Dafna D. Gladman ${ }^{15}$, Ken Kalunian ${ }^{19}$, Victoria P Werth ${ }^{20}$, Asad Zoma ${ }^{21}$, Sasha Bernatsky ${ }^{22}$, Munther Khamashta ${ }^{23}$, Søren Jacobsen ${ }^{24}$, Jill P. Buyon ${ }^{25}$, Mary Anne Dooley $^{26}$, Ronald van Vollenhoven ${ }^{27}$, Ellen Ginzler ${ }^{28}$, Thomas Stoll ${ }^{29}$, Christine Peschken ${ }^{30}$, Joseph L. Jorizzo ${ }^{31}$, Jeffery P. Callen ${ }^{32}$, Sam Lim ${ }^{33}$, Murat Inanc ${ }^{34}$, Diane L Kamen ${ }^{35}$, Anisur Rahman $^{9}$, Kristjan Steinsson ${ }^{36}$, Andrew G. Franks Jr ${ }^{37}$

${ }^{4}$ Department of Medicine, Division of Clinical Immunology and Rheumatology, University of Alabama at Birmingham, Birmingham, Alabama, USA 
${ }^{5}$ Rheumatology Research Group, Institute of Inflammation and Ageing, College of Medical and Dental Sciences, University of Birmingham, Birmingham, UK

${ }^{6}$ Department of Clinical Pharmacology, Oklahoma Medical Research Foundation, Oklahoma City, Oklahoma, USA

${ }^{7}$ Division of Rheumatology, CHU de Québec - Université Laval, Quebec City, Canada

${ }^{8}$ Arthritis Research UK Centre for Epidemiology, Centre for Musculoskeletal Research, Faculty

of Biology, Medicine and Health, The University of Manchester and NIHR Manchester

Biomedical Research Centre, Manchester University Hospital NHS Foundation Trust,

Manchester Academic Health Science Center, Manchester, UK

${ }^{9}$ Centre for Rheumatology Research, University College, London, UK

${ }^{10}$ Cedars-Sinai/David Geffen School of Medicine at UCLA, Los Angeles, California, USA

${ }^{11}$ Department of Clinical Sciences Lund, Section of Rheumatology, Lund University, Lund,

Sweden

${ }^{12}$ Northwestern University and Feinberg School of Medicine, Chicago, Illinois, USA

${ }^{13}$ Department of Rheumatology, Hanyang University Hospital for Rheumatic Diseases, Seoul,

Korea

${ }^{14}$ Division of Rheumatology, Department of Medicine and Department of Pathology, Queen

Elizabeth II Health Sciences Centre and Dalhousie University, Halifax, Nova Scotia, Canada

${ }^{15}$ Centre for Prognosis Studies in the Rheumatic Diseases, Toronto Western Hospital and

University of Toronto, Toronto Ontario, Canada

${ }^{16}$ Division of Rheumatology, Cumming School of Medicine, University of Calgary, Calgary, Alberta, Canada

${ }^{17}$ Feinstein Institute for Medical Research, Manhasset, New York, USA 
${ }^{18}$ Autoimmunity Institute, Allegheny Health Network, Pittsburgh, Pennsylvania, USA

${ }^{19}$ UCSD School of Medicine, La Jolla, California, USA

${ }^{20}$ Division of Dermatology, Hospital of the University of Pennsylvania and the Veteran's

Administration Medical Center, Philadelphia, Pennsylvania, USA

${ }^{21}$ Lanarkshire Centre for Rheumatology, Hairmyres Hospital, East Kilbride, Scotland, UK

${ }^{22}$ Division of Rheumatology, Department of Medicine, McGill University Health Centre, Montreal, Quebec, Canada

${ }^{23}$ Lupus Research Unit, The Rayne Institute, St Thomas' Hospital, King's College London School of Medicine, London, UK

${ }^{24}$ Copenhagen Lupus and Vasculitis Clinic, Centre for Rheumatology and Spine Diseases, Rigshospitalet, Copenhagen University Hospital, Copenhagen, Denmark

${ }^{25}$ Division of Rheumatology, Department of Medicine, New York University School of Medicine, New York, New York, USA

${ }^{26}$ Division of Rheumatology and Immunology, Department of Medicine, University of North Carolina, Chapel Hill, North Carolina, USA

${ }^{27}$ Amsterdam University Medical Centres, University of Amsterdam, Amsterdam, Netherlands;

Free University (VU) Amsterdam, Amsterdam, Netherlands; Amsterdam Rheumatology and Immunology Center, Amsterdam, Netherlands

${ }^{28}$ Department of Medicine, SUNY Downstate Medical Center, Brooklyn, New York, USA

${ }^{29}$ Department of Rheumatology, Kantonsspital, Schaffhausen, Switzerland

${ }^{30}$ Department of Medicine and Community Health Sciences, University of Manitoba, Winnipeg, Manitoba, Canada

${ }^{31}$ Department of Dermatology, Weill Cornell Medicine, New York, New York, USA 
${ }^{32}$ Department of Medicine, University of Louisville School of Medicine, Louisville, Kentucky USA

${ }^{33}$ Division of Rheumatology, Emory University School of Medicine, Atlanta, Georgia, USA

${ }^{34}$ Division of Rheumatology, Department of Internal Medicine, Istanbul Medical Faculty,

Istanbul University, Istanbul, Turkey

${ }^{35}$ Division of Rheumatology, Medical University of South Carolina, Charleston, South Carolina, USA

${ }^{36}$ Department of Rheumatology, Center for Rheumatology Research Fossvogur Landspitali

University Hospital, Reyjkavik, Iceland

${ }^{37}$ Division of Rheumatology, Department of Medicine, \& The Department of Dermatology, New York University School of Medicine, New York, New York, USA

The Hopkins Lupus Cohort is supported by NIH AR043727 and AR069572. 


\section{ABSTRACT}

Objective. Anti-beta 2 glycoprotein I IgA is a common isotype of anti-beta 2 glycoprotein I in SLE. Anti-beta 2 glycoprotein I was not included in the American College of Rheumatology (ACR) SLE classification criteria, but was included in the Systemic Lupus International Collaborating Clinics (SLICC) criteria. We aimed to evaluate the prevalence of anti-beta 2glycoprotein I IgA in SLE versus other rheumatic diseases. In addition, we examined the association between anti-beta 2 glycoprotein I IgA and disease manifestations in SLE. Methods. The dataset consisted of 1384 patients, 657 with a consensus physician diagnosis of SLE and 727 controls with other rheumatic diseases. Anti-beta 2 glycoprotein I isotypes were measured by ELISA. Patients with a consensus diagnosis of SLE were compared to controls with respect to presence of anti-beta 2 glycoprotein I. Among patients with SLE, we assessed the association between anti-beta 2 glycoprotein I IgA and clinical manifestations.

Results. The prevalence of anti-beta 2 glycoprotein I IgA was 14\% in SLE patients and 7\% in rheumatic disease controls (odds ratio, OR 2.3, 95\% CI: 1.6, 3.3). It was more common in SLE patients who were younger patients and of African descent $(\mathrm{p}=0.019)$. Eleven percent of SLE patients had anti-beta 2 glycoprotein I IgA alone (no anti-beta 2 glycoprotein I IgG or IgM). There was a significant association between anti-beta 2 glycoprotein I IgA and anti-dsDNA $(\mathrm{p}=0.001)$ and the other antiphospholipid antibodies $(\mathrm{p}=0.0004)$. There was no significant correlation of anti-beta 2 glycoprotein I IgA with any of the other ACR or SLICC clinical criteria for SLE. Those with anti-beta 2 glycoprotein I IgA tended to have a history of thrombosis (12\% vs $6 \%, \mathrm{p}=0.071)$, but the difference was not statistically significant.

Conclusion. We found the anti-beta 2 glycoprotein I IgA isotype to be more common in patients with SLE and in particular, with African descent. It could occur alone without other isotypes. 


\section{Introduction}

The IgA isotype of anti-beta 2 glycoprotein I (anti-B2-GPI) is the most common isotype in systemic lupus erythematosus (SLE) ranging between $20 \%$ and $59 \%$. (1-5) African-American SLE patients have a higher prevalence compared to other ethnic groups. $(6,7)$

Anti-beta 2 glycoprotein I IgA has been associated with thrombosis, particularly venous thrombosis, in SLE patients with secondary antiphospholipid syndrome (APS), as well as in patients with primary APS. $(1,8-11)$ It has also been associated with pregnancy related morbidity. $(12,13)$ However, IgA anti-B2-GPI is not included in the classification criteria of APS, partially due to the lack of uniform guidelines for the method of measurement and clinical cutoffs. (14)

Anti-beta 2 glycoprotein I IgA has been associated with clinical manifestations other than thrombosis, such as pulmonary hypertension and pulmonary fibrosis. (1) Anti-beta 2 glycoprotein I IgA was correlated with low complement C3, anti-Smith antibodies and a high erythrocyte sedimentation rate (ESR) in one SLE study. (1)

Anti-beta 2 glycoprotein I IgA is not included in the immunological criteria of the American College of Rheumatology (ACR) classification criteria for SLE (15), but it was included in the Systemic Lupus International Collaborating Clinics (SLICC) criteria. (16) Our aim was to assess the prevalence of anti-ß2-GPI $\operatorname{IgA}$ in the dataset that was used to derive and validate the SLICC SLE classification criteria. We also evaluated the sensitivity and specificity of anti-B2-GPI IgA to differentiate between those with SLE versus other rheumatic diseases, and to assess correlations of anti-ß2-GPI IgA with clinical and laboratory associations in SLE. 


\section{Methods}

The SLICC dataset consisted of clinical information on 1406 patients with rheumatic diseases from 25 international centers. These patient histories were collected in two phases: the "derivation" phase $(\mathrm{n}=716)$, and the "validation" phase $(\mathrm{n}=690)$. Lupus anticoagulant was determined by the individual sites. Sera for each patient from both phases were sent to RDL Reference Laboratory (Los Angeles, CA, USA) for measurement of immunologic markers. Anticardiolipin isotypes were measured by RDL Reference Laboratory using an enzyme immunoassay. The laboratory reference values were used for the cutoff. Anti-beta 2 glycoprotein I isotypes (IgG, $\operatorname{IgM}, \operatorname{IgA})$ were measured on the same samples as the other immunologic markers by ELISA assay at the laboratory of Joan Merrill, M.D. (Oklahoma Medical Research Foundation) for both phases. (17) The ELISA assay used beta 2 glycoprotein I as the antigen (Meridian Life Sciences, Memphis, TN, USA) and anti-human IgG, IgM or IgA alkaline phosphatase conjugate for detection (MilliporeSigma, St. Louis, MO, USA) The reference value was $<15$ with values $16-100$ considered positive. The reference value was set as 2 standard deviations above the mean of 30 healthy controls.

Using the clinical and laboratory data in the histories, patients from the derivation and validation phases were given a diagnosis of SLE or not based on a consensus process described previously. (16) Information on a patient's history of anti-B2-GPI and information on anti-ß32GPI results from the reference laboratory were included in the patient histories, if available. If either value was positive, anti-B2-GPI was scored as positive. Non-SLE patients consisted of rheumatoid arthritis, undifferentiated connective tissue disease, primary antiphospholipid antibody syndrome, vasculitis, chronic cutaneous lupus, scleroderma, Sjogren's syndrome, myositis, psoriasis, fibromyalgia, alopecia areata, and sarcoidosis. 
The research was reviewed and approved by the Johns Hopkins University School of Medicine Institutional Review Board (approval number NA_00039294). All study participants provided written informed consent.

\section{$\underline{\text { Statistical Methods }}$}

Subgroups of patients were compared with respect to the presence of anti-ß2-GPI and its isotypes. The statistical significance of observed differences was assessed using a Pearson Chi Square test. The associations between anti-ß32-GPI IgA and SLE disease manifestations, after adjusting for age and ethnicity, were assessed using multiple logistic regression.

\section{Results}

Of the 1406 patient histories, a consensus classification could not be reached for 14 histories, and information on anti-B2-GPI was missing on 8 patients. This analysis was based on the remaining 1384 patients (657 classified as SLE and 727 classified as non-SLE).

Of the 657 patients with SLE, 599 (91\%) were female, 394 (60\%) were Caucasian, 134 (20\%) were of African descent, and $76(12 \%)$ were Asian. Their mean age (years) at the time of assessment was $37.9(\mathrm{SD}=13.3)$. Of the 727 classified as not having SLE, $588(81 \%)$ were female, 530 (73\%) were Caucasian, 86 (12\%) were of African descent, and 48 (7\%) were Asian. Their mean age (years) at the time of assessment was $46.5(\mathrm{SD}=14.7)$

The prevalence of anti-ß2-GPI IgA in the central laboratory was 14\% (94/657) in SLE patients and 7\% (49/727) in controls $(\mathrm{p}<0.0001)$. (Table 1) Considering those positive for anti32-GPI IgA the median titer was somewhat higher among patients with SLE (median=29.5) than among controls (median=23.0, $\mathrm{p}=0.15$ for difference) . 
In contrast, there was no difference in the prevalence in anti-B2-GPI IgM or IgG in SLE patients versus controls (Table 1) vs. controls. Eleven percent (73/657) of SLE patients had antiß2-GPI IgA alone (no anti-32-GPI IgG or IgM).

The association between demographic characteristics and anti-B2-GPI IgA in patients with SLE is shown in Table 2. The highest prevalence of anti-B2-GPI IgA was found in African American SLE patients $(21.6 \%)$, compared to other ethnic groups $(\mathrm{p}=0.019)$. There was no difference in the prevalence of anti-B2-GPI IgA by sex. However, it was significantly more common in the younger age group $(\mathrm{p}=0.0048)$.

The association between the disease manifestations as defined in the ACR or SLICC classification criteria for SLE and anti-B2-GPI IgA in patients with SLE is shown in Table 3. There was a significant association between anti-B2-GPI $\operatorname{IgA}$ and anti-dsDNA ( $\mathrm{p}=0.0001)$ and the other antiphospholipid antibodies $(\mathrm{p}=0.0016)$. There was no significant correlation of antiB2-GPI IgA with any of the other ACR or SLICC clinical criteria for SLE.

We were able to evaluate the association between anti-ß2-GPI isotype with thrombosis and pregnancy morbidity in only half of the SLICC dataset patients (the derivation dataset). Those with anti-beta 2 glycoprotein I IgA tended to have a history of thrombosis (12\% vs 6\%, $\mathrm{p}=0.071$ ), but the difference was not statistically significant. Twenty-three patients had anti-beta 2 glycoprotein I IgA alone with no other antiphospholipid antibodies. Of those 1/23 (4\%) had a history of thrombosis. Of those in the derivation data set who did not have isolated anti-beta 2 glycoprotein I IgA, 28/285 (10\%) had a history of thrombosis. We did not have enough patients with adverse pregnancy outcomes to address an association with pregnancy loss.

If we removed anti-B2-GPI from the SLICC classification rule, the sensitivity of the rule (relative to physician classification) declined from $95.6 \%$ to $95.5 \%$, and the specificity changed 
from $88.3 \%$ to $88.4 \%$. The lack of change was due to the fact that it is only one component of antiphospholipid antibodies, which in turn is one component of the SLICC immunologic score.

\section{Discussion}

In the SLICC classification criteria dataset, only anti-ß32-GPI IgA was significantly more prevalent in SLE patients compared to other rheumatic disease patients. Anti-B2-GPI IgG and IgM were not significantly more prevalent in SLE patients than in those with other rheumatic diseases. The IgA isotype was the most frequent isotype, with 11\% of SLE patients having IgA alone without $\operatorname{IgM}$ and/or $\operatorname{IgG}$ isotypes.

Only the IgA isotype of SLE was associated statistically with the physician consensus diagnosis of SLE. Thus, the IgA isotype has particular relevance in SLE, but did not affect the overall sensitivity or specificity of the classification rule in any important way.

The variability in the prevalence studies of anti-ß2-GPI IgA in SLE patients may be partly attributed to the different ethnic composition of the studied population. The highest prevalence of anti-B2-GPI IgA is in African American SLE patients. (1,6,7) In our study, 22\% of the African descent patients had anti-ß2-GPI compared to $11 \%$ in patients of other ethnicities. Some variability might be due to different assays. A recent study by Tebo et al, found that the overall diagnostic and predictive value of anti-ß2-GPI IgA were dependent on the assays used. Assays were similar in predicting venous thrombosis, while there was a variable predictive result for pregnancy related morbidity. (13) Our study benefited from the use of only one central laboratory.

We found no significant association between anti-ß2-GPI IgA with ACR or SLICC classification criteria except for anti-dsDNA and other antiphospholipid antibodies. The association between anti-B2-GPI IgA and anticardiolipin antibodies has also been confirmed in 
other reports. $(3,4)$ In contrast, Lakos et al, found a significant correlation between anti- $32-\mathrm{GPI}$ IgA and thrombocytopenia and epilepsy. (5)

Mehrani et al, in a study based in the Hopkins Lupus Cohort, found a significant association of anti-ß32-GPI IgA isotype with pulmonary hypertension and pulmonary fibrosis. The IgA isotype of anti-B2-GPI was the only isotype to be significantly associated with antiSmith antibodies and elevated erythrocyte sedimentation rate in Hopkins SLE patients. (1)

In other studies, the IgA isotype of anti-32-GPI has been found to be associated with venous thrombosis. $(1,4,5)$ We did find an odds ratio of 2.5 in the SLICC dataset with an adjusted p-value of 0.065 .

Strengths of the study include that all assays were done in a central laboratory using the same assay, and that the international patient sample increased the generalizability of our findings. A limitation is the cross-sectional nature of the study. In conclusion, anti-ß2-GPI IgA is twice as frequent in SLE as in rheumatic disease controls and appears in $11 \%$ as the sole isotype. Thus, it could be missed if only $\operatorname{IgG}$ and $\operatorname{IgM}$ isotypes were checked; particularly important given the growing data that the $\operatorname{IgA}$ isotype is associated with thrombosis (13).

\section{Declaration of Conflicting Interests}

The authors declare that there is no conflict of interest. 


\section{References}

1. Mehrani T, Petri M, Escudero Contreras A, Khamashta MA, Hughes GR, Urowitz M. Association of IgA Anti-beta2 glycoprotein I with clinical and laboratory manifestations of systemic lupus erythematosus. J Rheumatol. 2011 Jan 1;38(1):64-8.

2. Fanopoulos D, Teodorescu MR, Varga J, Teodorescu M. High frequency of abnormal levels of IgA anti-beta2-glycoprotein I antibodies in patients with systemic lupus erythematosus: relationship with antiphospholipid syndrome. J Rheumatol. 1998 Apr;25(4):675-80.

3. Tsutsumi A, Matsuura E, Ichikawa K, Fujisaku A, Mukai M, Koike T. IgA class antibeta2-glycoprotein I in patients with systemic lupus erythematosus. J Rheumatol. 1998 $\operatorname{Jan} ; 25(1): 74-8$.

4. Lee SS, Cho ML, Joo YS, Kim WU, Hong YS, Min JK, et al. Isotypes of anti-beta2glycoprotein I antibodies: association with thrombosis in patients with systemic lupus erythematosus. J Rheumatol. 2001 Mar;28(3):520-4.

5. Lakos G, Kiss E, Regëczy N, Tarján P, Soltész P, Zeher M, et al. Isotype distribution and clinical relevance of anti-beta2-glycoprotein I (beta2-GPI) antibodies: importance of IgA isotype. Clin Exp Immunol. 1999 Sep;117(3):574-9.

6. Diri E, Cucurull E, Gharavi AE, Kapoor D, Mendez EA, Scopelitis E, et al. Antiphospholipid (Hughes') syndrome in African-Americans: IgA aCL and abeta2 glycoprotein-I is the most frequent isotype. Lupus. 1999 May 2;8(4):263-8.

7. Cucurull E, Gharavi AE, Diri E, Mendez E, Kapoor D, Espinoza LR. IgA anticardiolipin and anti-beta2-glycoprotein I are the most prevalent isotypes in African American patients with systemic lupus erythematosus. Am J Med Sci. 1999 Jul;318(1):55-60. 
8. Danowski A, Kickler TS, Petri M. Anti-beta2-glycoprotein I: prevalence, clinical correlations, and importance of persistent positivity in patients with antiphospholipid syndrome and systemic lupus erythematosus. J Rheumatol. 2006 Sep;33(9):1775-9.

9. Staub HL, Franck M, Ranzolin A, Norman GL, Iverson GM, von Mühlen CA. IgA antibodies to beta2-glycoprotein I and atherosclerosis. Autoimmun Rev. 2006 Dec;6(2):104-6.

10. Sweiss NJ, Bo R, Kapadia R, Manst D, Mahmood F, Adhikari T, et al. IgA anti-beta2glycoprotein I autoantibodies are associated with an increased risk of thromboembolic events in patients with systemic lupus erythematosus. Unutmaz D, editor. PLoS One. 2010 Aug 19;5(8):e12280.

11. Ruiz-García R, Serrano M, Ángel Martínez-Flores J, Mora S, Morillas L, Martín-Mola MÁ, et al. Isolated IgA Anti- $\beta 2$ Glycoprotein I Antibodies in Patients with Clinical Criteria for Antiphospholipid Syndrome. J Immunol Res. 2014;2014:1-8.

12. Lee RM, Branch DW, Silver RM. Immunoglobulin A anti- $\beta 2$-glycoprotein antibodies in women who experience unexplained recurrent spontaneous abortion and unexplained fetal death. Am J Obstet Gynecol. 2001 Sep;185(3):748-53.

13. Tebo AE, Willis R, Jaskowski TD, Guerra M, Pierangeli SS, Salmon J, et al. Clinical significance and correlations between anti- $\beta 2$ glycoprotein I IgA assays in antiphospholipid syndrome and/or systemic lupus erythematosus. Clin Chim Acta. 2016 Sep 1;460:107-13.

14. Miyakis S, Lockshin MD, Atsumi T, Branch DW, Brey RL, Cervera R, et al. International consensus statement on an update of the classification criteria for definite antiphospholipid syndrome (APS). J Thromb Haemost. 2006 Feb 1;4(2):295-306. 
15. Tan EM, Cohen AS, Fries JF, Masi AT, Mcshane DJ, Rothfield NF, et al. The 1982 revised criteria for the classification of systemic lupus erythematosus. Arthritis Rheum. 1982 Nov;25(11):1271-7.

16. Petri M, Orbai A-M, Alarcón GS, Gordon C, Merrill JT, Fortin PR, et al. Derivation and validation of the Systemic Lupus International Collaborating Clinics classification criteria for systemic lupus erythematosus. Arthritis Rheum. 2012 Aug;64(8):2677-86.

17. Dinu AR, Merrill JT, Shen C, Antonov I V, Myones BL, Lahita RG. Frequency of antibodies to the cholesterol transport protein apolipoprotein A1 in patients with SLE. Lupus. 1998 Jun 2;7(5):355-60. 
Table 1: Number (\%) with Anti-ß2-GPI by Diagnosis of SLE vs Non-SLE

\begin{tabular}{|lccc|}
\hline Anti-32-GPI subtype & SLE & Non-SLE & p- value \\
& $(\mathrm{n}=657)$ & $(\mathrm{n}=727)$ & \\
\hline IgA & $94(14 \%)$ & $49(7 \%)$ & $<0.0001$ \\
\hline IgM & $47(7 \%)$ & $38(5 \%)$ & 0.14 \\
\hline IgG & $42(6 \%)$ & $40(6 \%)$ & 0.48 \\
\hline
\end{tabular}


Table 2. Demographic characteristics and anti-32-GPI IgA in patients with SLE.

\begin{tabular}{|llcc|}
\hline & & Proportion $(\%)$ with & p-value \\
& & Anti-B2-GPI IgA & \\
\hline Ethnicity & African descent & $29 / 134(21.6)$ & 0.019 \\
& Caucasian & $45 / 394(11.4)$ & \\
& Asian & $14 / 76(18.4)$ & \\
& Other & $6 / 53(11.3)$ & \\
& Female & $85 / 599(14.2)$ & \\
& Male & $9 / 58(15.5)$ & \\
\hline Gender & $\leq 30$ & $40 / 200(20.0)$ & 0.0048 \\
& $>30$ & $53 / 455(11.7)$ & \\
\hline Age (years) & & & \\
& & & \\
& & &
\end{tabular}


Table 3. Number (Percent) of patients with a history of various SLE manifestations (as defined by the American College of Rheumatology (ACR) classification criteria for SLE) by anti-B2-GPI IgA in patients with SLE

\begin{tabular}{|c|c|c|c|c|c|}
\hline $\begin{array}{l}\text { Clinical and serologic } \\
\text { variables }\end{array}$ & $\begin{array}{c}\text { Anti-ß2-GPI } \\
\text { IgA }(\%)\end{array}$ & $\begin{array}{c}\text { Anti-ß2-GPI } \\
\operatorname{IgA}(\%)\end{array}$ & P-value & $\begin{array}{l}\text { Odds Ratio } \\
(95 \% \mathrm{CI})\end{array}$ & $\begin{array}{l}\text { Adjusted } \\
\text { P-value }\end{array}$ \\
\hline & $\begin{array}{l}\text { PRESENT } \\
(n=94)\end{array}$ & $\begin{array}{l}\text { ABSENT } \\
(\mathrm{n}=563)\end{array}$ & & & \\
\hline Malar Rash & $49(52.1 \%)$ & $245(43.5 \%)$ & 0.12 & $1.4(0.9,2.2)$ & 0.16 \\
\hline Discoid Rash & $19(20.2 \%)$ & $101(17.9 \%)$ & 0.60 & $1.3(0.7,2.2)$ & 0.42 \\
\hline Photosensitivity & $46(48.9 \%)$ & $287(51.0 \%)$ & 0.71 & $1.0(0.6,1.5)$ & 0.91 \\
\hline Oral Ulcers & $36(38.3 \%)$ & $234(41.6 \%)$ & 0.55 & $0.9(0.6,1.4)$ & 0.57 \\
\hline Arthritis & $62(66.0 \%)$ & $371(65.9 \%)$ & 0.99 & $1.0(0.6,1.6)$ & 0.93 \\
\hline Pleurisy & $32(34.0 \%)$ & $143(25.4 \%)$ & 0.079 & $1.4(0.9,2.3)$ & 0.16 \\
\hline Pericarditis & $12(12.8 \%)$ & $61(10.8 \%)$ & 0.58 & $0.9(0.5,1.9)$ & 0.86 \\
\hline Proteinuria & $35(37.2 \%)$ & $169(30.0 \%)$ & 0.16 & $1.1(0.7,1.8)$ & 0.71 \\
\hline Seizure & $5(5.3 \%)$ & $31(5.5 \%)$ & 0.94 & $1.0(0.4,2.6)$ & 0.92 \\
\hline Psychosis & $3(3.2 \%)$ & $11(2.0 \%)$ & 0.44 & $1.5(0.4,5.8)$ & 0.54 \\
\hline Hemolytic Anemia & $8(8.5 \%)$ & $34(6.0 \%)$ & 0.36 & $1.3(0.53,2.9)$ & 0.59 \\
\hline Leukopenia & $33(35.1 \%)$ & $160(28.4 \%)$ & 0.19 & $1.2(0.7,1.9)$ & 0.56 \\
\hline
\end{tabular}




\begin{tabular}{|c|c|c|c|c|c|}
\hline $\begin{array}{l}\text { Clinical and serologic } \\
\text { variables }\end{array}$ & $\begin{array}{c}\text { Anti-ß2-GPI } \\
\operatorname{IgA}(\%) \\
\text { PRESENT } \\
(\mathrm{n}=94)\end{array}$ & $\begin{array}{c}\text { Anti-ß2-GPI } \\
\operatorname{IgA}(\%) \\
\text { ABSENT } \\
(\mathrm{n}=563)\end{array}$ & P-value & $\begin{array}{l}\text { Odds Ratio } \\
(95 \% \mathrm{CI})\end{array}$ & $\begin{array}{l}\text { Adjusted } \\
\text { P-value }\end{array}$ \\
\hline Lymphopenia & $28(29.8 \%)$ & $174(30.9 \%)$ & 0.83 & $0.9(0.6,1.5)$ & 0.72 \\
\hline Thrombocytopenia & $17(18.1 \%)$ & $79(14.0 \%)$ & 0.30 & $1.2(0.7,2.3)$ & 0.46 \\
\hline Anti-dsDNA $^{1}$ & $77(81.9 \%)$ & $334(59.3 \%)$ & $<0.0001$ & $3.0(1.7,5.4)$ & 0.0001 \\
\hline Anti-Smith $^{1}$ & $37(39.4 \%)$ & $140(24.9 \%)$ & 0.0034 & $1.5(0.9,2.5)$ & 0.085 \\
\hline $\begin{array}{l}\text { Antiphospholipid } \\
\text { Antibodies }^{1}\end{array}$ & $71(75.5 \%)$ & $315(56.0 \%)$ & 0.0004 & $2.3(1.4,3.8)$ & 0.0016 \\
\hline Anticardiolipin $\operatorname{IgG}^{1}$ & $42(44.7 \%)$ & $157(26.1 \%)$ & 0.0002 & $2.2(1.4,3.5)$ & 0.0009 \\
\hline Anticardiolipin IgM ${ }^{1}$ & $35(37.2 \%)$ & $105(18.7 \%)$ & $<0.0001$ & $2.6(1.6,4.2)$ & 0.0001 \\
\hline Anticardiolipin $\operatorname{Ig} \mathrm{A}^{1}$ & $10(10.6 \%)$ & $13(2.3 \%)$ & $<0.0001$ & $5.8(2.3,14.9)$ & 0.0002 \\
\hline Lupus Anticoagulant $^{2}$ & $26(28.6 \%)$ & $72(13.2 \%)$ & 0.0002 & $2.5(1.4,4.3)$ & 0.0012 \\
\hline Anti-ß32-GPI IgG ${ }^{2}$ & $11(11.7 \%)$ & $31(5.5 \%)$ & 0.023 & $3.2(1.5,7.0)$ & 0.0034 \\
\hline Anti-ß2-GPI IgM ${ }^{2}$ & $14(14.9 \%)$ & $33(5.9 \%)$ & 0.0017 & $3.3(1.6,6.8)$ & 0.0011 \\
\hline $\begin{array}{l}\text { History of } \\
\text { thrombosis }{ }^{3}\end{array}$ & $9(12.3 \%)$ & $14(6.0 \%)$ & 0.071 & $2.5(0.9,6.4)$ & 0.065 \\
\hline
\end{tabular}

${ }^{1}$ For these variables, the patients were considered positive if they had a clinical history of the serologic marker, or if a serologic specimen collected specifically for the parent study was positive for the marker.

${ }^{2}$ For these variables patients were considered positive based only on the serologic specimen collected specifically for the parent study. 
${ }^{3}$ Based only on the patients in the derivation data set (73 positive for Anti-B2-GPI IgA, and 235 negative for Anti-32-GPI IGA)

${ }^{4}$ P-value adjusted for Ethnicity and Age 